\title{
Slicing in Locavore Infrastructures
}

\author{
Glenn Ricart \\ US Ignite and U. Utah \\ $115018^{\text {th }}$ St NW, Suite 900 \\ Washington, DC 20036 \\ +1 703-942-9171 \\ Glenn.Ricart@us-ignite.org
}

\begin{abstract}
A Locavore Infrastructure is one which has all of its elements in high-bandwidth and low-latency proximity. It typically combines edge computing elements with an adjacent access network. The growing number of communicating devices and things creates a large and often steady demand for collecting and integrating local information in a Locavore Infrastructure. Slices of this infrastructure can provide architectural advantages in security, meeting performance expectations, and billing. Dynamic slices can provide some of the same kinds of surge capabilities for which traditional cloud computing is prized. Slices can be implemented using a variety of orchestration techniques.
\end{abstract}

\section{Categories and Subject Descriptors}

- Computer systems organization Real-time system architecture - Computer systems organization Cloud computing - Computer systems organization Client-server architectures

\section{General Terms}

Performance, Design, Economics, Reliability, Security.

\section{Keywords}

Locavore, edge, slices, slicing, edge security, Internet of Things, infrastructure hypervisor.

\section{INTRODUCTION}

Cloud computing and its ability to provide elastic resources has compelling reasons to move toward network edges. These edges are close to the source of increasing amounts of data as the Internet of Things threatens to overrun the Internet of people - at least in numbers if not also in amount of data generated [7]. Computing at the edges can reduce response time for the growing number of applications and services that beneficially operate in

Permission to make digital or hard copies of all or part of this work for personal or classroom use is granted without fee provided that copies are not made or distributed for profit or commercial advantage and that copies bear this notice and the full citation on the first page. Copyrights for components of this work owned by others than the author(s) must be honored. Abstracting with credit is permitted. To copy otherwise, or republish, to post on servers or to redistribute to lists, requires prior specific permission and/or a fee. Request permissions from Permissions@acm.org.

$D C C^{\prime} 16$, July 25-28 2016, Chicago, IL, USA

Copyright is held by the owner/author(s). Publication rights licensed to ACM.

ACM 978-1-4503-4220-9/16/07 ...\$15.00

DOI: http://dx.doi.org/10.1145/2955193.2955207 close synchrony with people and the real world in which they live [16]. What is lost in economy of scale is often overwhelmed by the requirement to be more responsive or to reduce backbone transit costs.

As clouds move toward the edge, they must still run and isolate multiple applications and services in the same cloud. Given the typically smaller sizes of edge clouds and reduced response time demands, effective dynamic orchestration takes on added importance.

This paper discusses slicing as a means of providing dynamic multi-tenancy in edge infrastructure. While edge clouds and access networks may not have the same economy of scale as massive datacenters, they do have the advantage of being potentially more nimble in optimizing the use of their resources. Co-optimizing the edge computing and the access network infrastructure also becomes possible.

\section{LOCAVORE INFRASTRUCTURE}

A Locavore Infrastructure is one which has all of its elements in high-bandwidth and low-latency proximity as viewed by the applications it runs [12]. The name comes from the term for people who preferentially consume foods grown locally [17] In the computing case, locavore infrastructure preferentially handles local data and tasks. A locavore infrastructure is typically edge computing combined with a high-bandwidth and low-latency access network. In this respect, it's closely related to edge computing, fog computing [3], dew computing [XX], and cloudlets [13] but with a co-optimized access network explicitly included.

In traditional remote clouds, requests and data are moved to a remote data center where they are acted upon and results are returned. The data are moving to a compute-cost-efficient datacenter facility. Costs and delays in the network are assumed to be overwhelmed by the efficiencies of scale in CPU, memory, and data storage. In a locavore environment, a portion of the data center is moving to the data and to its users.

Coupling edge computing with an access network allows for co-optimization of the compute and storage environment and the network paths involved. For example, a medical monitoring application that demands a very high degree of responsiveness can be given dedicated or priority compute and storage resources while packets being sent to or from the application can be given priority on the network. Similarly, a time-insensitive application could be given scavenger priority on both the local compute/storage facility as well as on the network. More methods of co-optimization and federation are discussed in later sections. 
A locavore infrastructure has several advantages over traditional clouds:

a. Applications and services can be more responsive because they are not as affected by the speed of light and hop count delays needed to reach traditional remote clouds.

b. Information which can be aggregated, processed, or disseminated locally can skip the Internet backbone entirely, and that helps to manage the growth of backbone traffic.

c. More traffic is kept local and therefore is not subject to failures elsewhere. School testing throughout Alaska was cancelled due to a fiber break in Kansas [9].

d. Greater bandwidth end-to-end is often available because fewer aggregation points are encountered. If the compute and storage elements are located at the head-end of the access provider, for example, bandwidth used by applications is not limited by having to pass through peering points or aggregation points upstream of the locavore infrastructure.

e. Access service providers may have lower costs because the locally-handled portion of the traffic does not have to travel one of the Internet backbones. A local ISP has no cost for handing off the traffic to a tier 1 provider if it instead processes it locally.

f. Massive scale-out of functions provided on a per-user basis is easily achieved by spreading the scale-out over many locavore edges.

There are several interesting places where the edge computing and storage meets the access network. Perhaps easiest to grasp is a compute-storage facility well-connected at the headend of a city-wide ISP. It meets the definition of all elements being in high-bandwidth and low-latency proximity. Note that the access network could be wireless.

In addition to cellular ISPs, the same pattern of locavore architecture can be replayed at a wireless access point. In the Paradrop [18] architecture, "chutes" (small applications) are executed locally within the wireless router and have high bandwidth (up to multi-gigabit on $802.11 \mathrm{ac}$ wireless routers) and low-latency access to the wireless device. The wireless router can be directed to give critical applications priority access to both the CPU and to the radio to co-optimize the responsiveness of the critical application.

A smartphone with radio connections (typically Bluetooth or $\mathrm{WiFi}$ ) can also function as locavore infrastructure and co-optimize compute and storage and network.

\section{SLICING}

A slice is a time- and/or space-division allocation of resources devoted to a particular purpose.

Compute, memory and storage slicing have a long history beginning at least as early as timesharing [10]. Some early CPU schedulers relied on time-slicing for multi-tasking [10]. Virtual memory can be used to implement memory slicing and to allow virtual memory addresses to be repeated as long as they are in different slices. Several fairly static storage slicing techniques are in wide use including disk partitions and virtual storage volumes.

A popular way of slicing cloud infrastructure is with the use of virtual machines (VMs) and virtual storage volumes. A hypervisor [4] is used to share a single physical component as multiple virtual versions. Typically hypervisors attempt to provide not only namespace isolation but also at least a degree of performance isolation. Containers are also increasingly popular for namespace isolation.

Several slicing mechanisms also exist for networks and network traffic. These include VLANs. MPLS, SPB [6], SDNdefined flow segregation [14], and tunneling. These techniques provide varying degrees of isolation in addressing space and performance and are often implemented through virtualization. Typically the slice information is added by the network based on the physical path by which the traffic arrives. In VLANs, an 802.1Q VLAN tag is added. In MPLS, a label is added. In SPB, a logical Ethernet is operated over the physical layer and either packets are encapsulated within a logical MAC address $(802.1 \mathrm{~h})$ or given an $802.1 \mathrm{Q} / 801.2 \mathrm{ad}$ tag and delivered only to other members of the logical network. In SDN-defined flow segmentation, matches of certain tags, addresses, and other characteristics of the header trigger different handling so that the packet is only delivered according to the rules for that flow. In tunneling, the original packet is encapsulated in another which carries it only to another member of the logical network. In all these cases, the original layer 3 and layer 2 addresses can be duplicated between slices because other information is used to keep the packet within its own slice.

The benefits of slicing include:

a. Isolation between slices including the ability to duplicate address spaces. A degree of security is provided when it's impossible to directly address information in a different slice.

b. Different slices can be given different levels of service.

c. Slices allow for more rapid and dynamic "reconfiguration" of the network infrastructure.

d. The use of the resource(s) within a slice can be measured and reported on a per-slice basis.

e. Error conditions in one slice need not affect other slices (unless there is a physical layer issue which affects all slices using that physical layer).

GENI [5] uses these benefits to allow projects and students to create their own slices and experiment with them while not having serious impact on other slices. In GENI, the slices have expiration dates so that slice resources, even when virtual, can be reclaimed even if the user who created them takes no further action.

\section{APPLICATION OR SERVICE SLICES}

An application or service slice is a set of resources hosting an application, a service, or an application or service instance. This will typically include a set of sub-slices for compute/memory resources, storage resources, and network resources.

Providing a combination of VM sub-slices, storage subslices, and interconnecting network sub-slices as a single experiment slice appears to be an innovation of GENI - The Global Environment for Network Innovation [5]. The sub-slices are called "slivers" in GENI. They are first allocated individually at one or more facilities and then packaged as a service slice and delivered to the user for their experiment. 
The benefits are similar to those of slicing, but now they apply to applications and services.

a. Isolation between applications and services. For example, applications which need different versions of libraries can have their own versions of the libraries loaded into their own slice without affecting other applications.

b. Different applications and services can be given different levels of service.

c. Slices can be given more or fewer resources as needed to accommodate application or service demand. Similarly, littleused applications or services can be "spun-up" when needed by creating a new slice. Conversely, applications or services can be "spun-down" by optionally capturing state and then de-allocating the slice.

d. The use of the application or service can be measured and reported on a per-application or per-service basis, and form the basis for billing applications for the resources they use.

e. Error conditions in one application or service don't affect others.

Two forms of application or service slices are possible if the application or service might have more than one user.

In the slice-per-service version, the application or service has its own slice, regardless of the number of users it serves. It internally multiplexes each of its users and can internally decide how to best use the resources of its slice in serving its users. Isolation between users is the responsibility of the service itself. The application or service itself must ask for its slice to be enlarged or shrunk to efficiently deal with variations in demand.

In the slice-per-user version, each user is given their own slice instantiating the application or service. In that case, the slice provides the isolation between users, and a service failure for one user will not affect any of the other users. It is possible to give different users different levels of service for the same application by the way their different slice resources are managed. Slice usage can be attributed to and billed to a specific user. However, slice overhead is multiplied and slice efficiency becomes a key consideration.

\section{APPLICATIONS ENABLED BY LOCAVORE SLICES}

Applications or services running on a sliced locavore infrastructure enjoy both the advantages of the locavore approach and the benefits of slicing. The result can enable powerful new classes of applications and services that may not be feasible to run on today's commercial cloud infrastructure and commercial Internet access services.

Some examples include:

- Streaming VR to reduce the cost of VR/AR for the underserved. If the model and graphics processing is performed in an edge or locavore server, a gigabit access stream of video to the headset reduces the cost of the headset to perhaps $\$ 100+$ smart phone. Head motions must very rapidly pan the viewed image to change to avoid confusing the brain. The latency window is $10-$ $20 \mathrm{msec}$. total latency according to Occulus CEO Brendan Iribe [8].
- Streaming visualization of very large datasets too large to keep in the end-user device. One example of this is the Pollution Visualizer [2] which allows a user to twist a knob to change the date for the pollution map while also allowing smooth zoom and pan.

- Natural local videoconferencing multiplexer. If latency is reduced in video conferencing, its usefulness might possibly be increased by an order of magnitude.

- Neighborhood smart grid coordination and other similar cyber-physical (CPS) systems with low latency requirements. While a building or campus tends to have a "head end" already to synchronize the grid across buildings in a campus, a neighborhood tends to need a local coordination resource (such as a locavore infrastructure would provide) to do this. It's particularly important if there is net inflow from some parties such as solar cell power being net metered.

- Intensive home medical monitoring. Sometimes people are kept in the hospital just so they can be instrumented to track the functioning of various internal organs and systems. With sufficiently responsive locavore infrastructure and sufficiently private slices, it will be feasible to do hospital-quality monitoring from the patient's own home, greatly reducing healthcare costs.

- Making sense from multiple sensors. Is the temperature rising on one side of the house because of sun loading? Or does the pattern of temperatures look more like a small fire growing? Are the light-scattering atmospheric sensors also detecting an increase in small particles which scatter light (even though the threshold isn't enough for them to trip independently)?

\section{IMPLEMENTATION CHALLENGES}

New fiber, copper, and wireless technologies are adding significant access bandwidth. Within the past decade, the offered speeds for home broadband have increased by two orders of magnitude, primarily thanks to the new competition for gigabit performance offered first by Google Fiber and later copied by most access providers [11]. Applications and services which were previously bandwidth-limited are generally no longer limited on gigabit access networks. In the wireless world, $5 \mathrm{G}$ promises the same kind of uptick in capability.

The bottleneck in locavore infrastructure is therefore expected to be the compute/storage infrastructure. Utilization in locavore infrastructure doesn't enjoy the same smoothing that the law of large numbers supplies in very large datacenters. Hence, application and service allocation and placement become far larger issues. Internal and external fragmentation of all resources (except perhaps gigabit network) are likely to become important performance limitations.

If the system is providing low-latency performance on demand, there is less ability to shuffle the resources supplying a slice while that slice is running. That implies that correct selection of resources for a slice becomes more important.

\section{RESOURCE ALLOCATION}

To begin execution of an application or service within a slice, one must allocate the resources for the slice, interconnect them, initialize them, and connect the slice to its externalities such as the Internet via virtual NICs or other services such as billing services. 
The factors that might be considered in deciding which virtual resources might be allocated to a slice include:

a. Locality, if the slice needs low-latency or very high bandwidth;

b. Capacity of the underlying physical resource to accommodate the workload expected to be presented by this slice;

c. Proximity to other slice virtual resources (to avoid having to add additional network links to haul intermediate processing);

d. Price of this virtual resource, considered variously in terms of money, latency, reduction in redundancy, reduction in reliability, opportunity cost, similarity to other slices on the same physical resource, etc.

e. Matching the capacity of this portion of the slice to keep it in balance with other portions of the slice. For example, spending compute time on $4 \mathrm{~K}$ quality graphics is not warranted if the network capability assignable to the slice will only support HD graphics.

To maintain low-latency real-time-like performance, we must not over-commit the physical resources supporting the virtual resources allocated to the slice. Or, for systems running workloads of both time-critical and non-time-critical applications and services, the hypervisor supporting the virtual resources allocated to slices will need to understand the varying performance requirements of the slices and treat the virtual resources differently bearing in mind the needs of the workload running in each slice. There is considerable additional research needed in this area.

\section{LOCAVORE SLICE MANAGEMENT}

A number of options are available for mechanisms to manage locavore slices. GENI uses a manifest-specification created by the user using a tool to pre-allocate the resources declared to be necessary. This works well for applications with a known resource utilization profile or to set up a best guess initial slice. During execution, the slice itself may realize it is underprovisioned or over-provisioned and request additional resources be added to the slice, or the slice itself may turn back some resources it already had obtained. This permits expansion and contraction of the slice resources in traditional cloud fashion. Similarly, an orchestration layer may recognize the slice resources might be usefully augmented or reduced.

Given that the compute cores are expected to be one bottleneck in sliced locavore infrastructure, explicit time slicing of the cores (implemented by a hypervisor) may be used to manage resources to support a locavore slice. Time slices not being utilized might be distributed among active slices.

Of course, in addition to optimization of slice execution, there may be external factors which trump optimization concerns, including: (a) Public safety and communications priorities, (b) Manual scheduling for one-of-a-kind resources (LHC detectors at CERN, etc.), (c) Sandboxing new versions of applications, (d) Proximity to external interfaces or other externalities, (e) Ability to take and keep snapshots for longer-running applications, and (f) Facilitating slice mobility.

Within a physical resource, there may be concerns about information leakage between virtual machines due to such things as timing channels. For highly sensitive traffic, additional segregation at the physical resource level may be wise.

\section{SMART CONNECTED COMMUNITIES}

As the Internet's main load moves from supporting people and their browsers toward supporting an explosion of data and smart devices, we expect the need for Locavore Slices to also grow rapidly.

In smart community sensor environments, it is often not the reading of one sensor at one time that's valuable but rather the changes in readings of multiple sensors in the same neighborhood and served by the same locavore slice compared to each other and over time. The logical place to aggregate and provide first-level analysis is at the edge and near the sensors - that is, in a locavore slice.

In cyber-physical systems (CPS), typically local sensors generate information which is analyzed to trigger local actions. When response time from sensing to action must be minimized, a locavore slice may provide the needed response time while minimizing possible network failure points. When response time isn't critical, a locavore slice still keeps local traffic and decisions local.

In smart and connected communities, keeping local traffic local is often valued to prevent local problems due to distant failures.

\section{RELATIONSHIP TO NFV}

Network functions themselves are often best performed at the edge (e.g., subscriber traffic measurement), and architecturally, edge-based network function virtualization (NFV) is very similar to locavore slicing. Both are placed at the edge, can be parts of chained services, and provide for massive scaleout.

An important use case is the use of NFV in supporting wireless infrastructure at the wireless edge. While NFV supports the wireless operator's function, a similar compute/storage box sitting at the same place in the wireless network function chain could provide user-specified services and provide added value.

Orchestration of NFV and orchestration of slices appear to be very similar problems. However, the ownership and optimization could be quite different. In NFV, the owner is the network operator. In locavore slices, the owner may be the network operator, but the owner may also be a different service provider. In addition, NFV functions are often deployed as part of fairly static configurations allowing fairly static resource allocations. In locavore slices, we anticipate highly dynamic slices with slices coming and going as fast as instances of individual applications.

However, even though there are differences, a good starting point for locavore research is existing NFV research.

\section{FEDERATION}

The most complex form of interaction between locavore infrastructures is one where different edge facilities are owned by different administrators and may furthermore have differing capabilities and protocols. Interoperation under these circumstances requires federation of multiply-administered locavore infrastructures. 
The additional challenges include:

a. Potentially differing specifications and mechanisms for slice construction and allocation

b. Potentially different semantics for the locavore slices

c. Challenges in authentication, authorization, service management, optimization, and billing

d. Potential problems in sharing state information (both technical information and business information) between administrative boundaries

Federation is also a considerable research challenge, but practical progress can be made by simplifying the expected interaction across administrative boundaries. If only data needs to be exchanged (e.g., as over a peering point), we have many examples of how this can be accomplished.

As noted in the slicing section of this paper, the actual mechanism of slicing often involves either encapsulating network packets in slice envelopes, or, prepending (or appending) a sliceidentification tag of some sort. The format and size and contents of the tag or encapsulation information varies from MPLS to VLAN to VxLAN to an OpenFlow flow, but an important observation is that if the packet is not already obviously in one slice or another, one prepends and/or appends additional information to facilitate handling.

In the case of federation, one may need to transliterate the information that identifies the slice to which a packet belongs to another form understood by the federated system.

But that is the easy part. How does an application or service being instantiated across multiple federated domains tell each administrative domain, in its own language, the slice requirements for setting up the portion of the slice in each administrative domain as well as telling gateways about the transliterations needed? And as the slice runs and needs more or fewer or different resources, how does it do that in an inter-domain way? How are issues such as time criticality handled?

There appear to be three directions for solution, all worthy of additional research. In a brokered solution, the broker is contacted to organize the details of the multi-domain slice. The broker talks the languages of all the domains, and executes the appropriate mechanisms for creating individual slices in each administrative domain and instantiating compatibility services to interconnect them. This itself may be regarded as a broker service-an inter-domain service. Note that the inter-domain broker service may have no control at all over the administrative domains but may appear purely as a user of the domain's service. For example, the stitching service in GENI operates in this fashion.

In a peer coordinated solution, a slice in one administrative domain knows enough about other administrative domains to which it might connect that it can "reach across" to the other administrative domain to ask it to add a corresponding slice and, if needed, a transliteration that connects the slices. It may get this information from published descriptions. In this model, each slice starts in a single, parent, administrative domain and makes arrangements to extend itself by learning the language of other administrative domains.
Finally, in an agent solution, administrative domains that may want to interoperate with each other contain agents representing foreign administrative domains. Those agents talk the local slice language and extend the slice using mechanisms, identifications, and authorizations native to the local slice. However, the agents provide the transliterations and other services needed to make the slice appear native in each domain.

\section{LOCAVORE HIERARCHIES}

Clouds can be usefully stacked hierarchically (e.g., Cloud, Fog, Dew) [15]. Similarly, Locavore Infrastructures can also be stacked hierarchically to more efficiently serve large distributed applications or to improve their economics.

Consider the streaming VR Application from Section 5 which requires a 10-20 msec. response time. A Locavore Infrastructure might be placed at the head-end of a city-wide access network or at a city Locavore exchange point in order to meet the response time requirement.

Now consider the same application for a classroom of students with VR headsets. A 360-degree sphere of vision could be sent from the head-end to a lower locavore tier, perhaps implemented in a wireless access point, that coordinates $\mathrm{WiFi}$ within the classroom and sends each student their own 3D view field. This tiered or recursive locavore approach helps to keep the bandwidth required to a gigabit. Rick McGeer has a paper pending on this application in which each locavore layer has an edge cloud and an access network co-optimized.

\section{BUSINESS CONSIDERATIONS}

If a locavore slice infrastructure is good for smart and connected communities, what business entity or entities will implement them? There appear to be several choices.

One possibility is that local access carriers may implement locavore infrastructure at their head-ends as value-added services. Given that the access carriers almost surely have NFV infrastructure at the head-ends already, an extension of the NFV infrastructure could be turned over to execute applications services. The value of such services might be billed to the service or application provider so that the user pays a single fee to the service or application provider.

Another possibility is that datacenter operators may decide that locavore clouds are a natural extension of their services when the customer needs larger bandwidth or better response time than their normal clouds can provide. Several authors predict a boom in so-called micro data centers which can be placed close to Internet of Things infrastructure [1]. If services are provided to other applications and services, a business relationship would have to be established with the provider of the other applications and services. It may be difficult to do so if the other applications and services are considered competitive and that may limit this option.

Yet another possibility is that in open-access systems where local access and ISP services are separated, the ISP may provide locavore infrastructure services.

And, of course, nothing prevents new market entrants from providing competitive locavore slices and services. 


\section{CONCLUSION}

The US Ignite organization is encouraging the development and deployment of new Internet applications that may require locavore-provided services to meet bandwidth and/or response time requirements. The examples given in Section 5 come from existing and potential US Ignite projects. In addition, slicing may add important additional security and isolation for applications in healthcare and finance. The provision of competitive locavore infrastructure services with federation may well be necessary to create a robust and interoperable locavore slicing architecture to support smart and connected community applications.

\section{ACKNOWLEDGMENTS}

These implications of slicing in locavore infrastructures have been developed in discussions with many others in the US Ignite family but principally with Rick McGeer. The work was partly supported by award 1531046 from the National Science Foundation.

\section{REFERENCES}

[1] Aazam, Mohammad, and Eui-Nam Huh. 2015. Fog computing micro datacenter based dynamic resource estimation and pricing model for IoT." Advanced Information Networking and Applications (AINA), 2015 IEEE 29th International Conference on. IEEE, 2015.

[2] Bhojwani, Sushil, et al. 2015. The Ignite Distributed Collaborative Scientific Visualization System. IEEE 7th International Conference on Cloud Computing Technology and Science (CloudCom). IEEE, 2015.

[3] Bonomi, Flavio, et al. 2012. Fog computing and its role in the internet of things. Proceedings of the first edition of the MCC workshop on Mobile cloud computing. ACM, 2012.

[4] Chen, Peter M., and Brian D. Noble. 2001. When virtual is better than real [operating system relocation to virtual machines]. Hot Topics in Operating Systems, 2001. Proceedings of the Eighth Workshop on. IEEE, 2001,

[5] Elliott, Chip. 2008. GENI-global environment for network innovations. LCN. 2008.

[6] Finn, Norman. 2005. Shortest Path Bridging. Sep 22 (2005): $1-95$.
[7] Greenough, John. 2015. The Internet of Everything 2015, Business Insider Intelligence, produced by Adam Thierer and Andrea Castillo, Mercatus Center at George Washington University, 2015.

[8] Iribe, Brendan. Private conversation, April 29, 2016.

[9] Lunau, Kate. 2016. Alaska Cancels School Tests After a Fiber Optic Cable Was Cut in Kansas, reported at http://motherboard.vice.com/read/alaska-cancels-schooltests-after-a-fiber-optic-cable-was-cut-in-kansas, April 6, 2016.

[10] Meyer, Richard A., and Love H. Seawright. 1970. A virtual machine time-sharing system. IBM Systems Journal 9.3 (1970): 199-218.

[11] Pak, Steve. 2015. Comcast Bolts Past Google Fiber, AT\&T GigaPower with New Gigabit Pro 2Gbps Internet Service, Yabada at http://en.yibada.com/articles/24187/20150403/comcast-boltspast-google-fiber-t-gigapower-new-gigabit-pro.htm, April 3, 2015.

[12] Ricart, Glenn. 2013. US Ignite Network Architectures, TIP 2013 conference (one of the Joint Techs series), Honolulu, HI, January 16, 2013.

[13] Satyanarayanan, Mahadev, et al. 2009. The case for vmbased cloudlets in mobile computing. Pervasive Computing, IEEE 8.4 (2009): 14-23.

[14] Seddiki, M. Said, et al. 2015. FlowQoS: Per-Flow Quality of Service for Broadband Access Networks. (2015).

[15] Skala, Karolj, et al. 2015. Scalable distributed computing hierarchy: Cloud, fog and dew computing. Open Journal of Cloud Computing (OJCC) 2.1 (2015): 16-24.

[16] SmartFuture2015. 2015. Retrieved from demos tab of www.smartfuture2015.com, March 2015.

[17] The Local Foods Wheel. 2005. Retrieved from http://www.localfoodswheel.com/locavores/, August 2005.

[18] Willis, Dale F., Arkodeb Dasgupta, and Suman Banerjee. 2014. Paradrop: a multi-tenant platform for dynamically installed third party services on home gateways. Proceedings of the 2014 ACM SIGCOMM workshop on Distributed cloud computing. ACM, 2014. 